\title{
Erratum
}

\section{Small scale spatial heterogeneity influences predation success in an unexpected way: Model experiments on the functional response of predatory mites (Acarina)}

Kaiser $\mathrm{H}$

Oecologia (Berlin) (1983) 56:249-256

1. Substitute Fig. 3 (p. 251) for Fig. 6 (p. 254) and vice versa; the captions are already in the correct places.

2. Fig. 4 has been reversed. In the printed form the columns should therefore read c, b, and a. 